Arq. Bras. Med. Vet. Zootec., v.69, n.1, p.48-56, 2017

\title{
Avaliação hematológica e hemogasométrica de potros nascidos de éguas com placentite ascendente
}

\author{
[Hematological and hemogasometric evaluation of foals born from mares \\ with ascending placentitis] \\ R.S. dos Santos ${ }^{1}$, M.N. Corrêa ${ }^{1}$, L. O. de Araújo $^{1 *}$, F.M. Pazinato ${ }^{1}$, L.S. Feijó ${ }^{1}$, B.R. Curcio ${ }^{1}$, \\ R.C. Ferreira ${ }^{2}$, C.E.W. Nogueira ${ }^{1}$ \\ ${ }^{1}$ Faculdade de Veterinária - Universidade Federal de Pelotas - FV-UFPel - Pelotas, RS \\ ${ }^{2}$ Hospital Escola - Universidade Federal de Pelotas - HE-UFPel - Pelotas, RS
}

\begin{abstract}
RESUMO
A placentite é a principal causa de partos prematuros, aborto e nascimento de potros comprometidos, podendo causar hipóxia e septicemia. A hematologia e a gasometria venosa fornecem informações importantes para o monitoramento da saúde de potros nascidos de éguas com placentite. O objetivo deste trabalho foi descrever os valores hematológicos e hemogasométricos durante as primeiras $24 \mathrm{~h}$ de vida em potros nascidos de éguas mestiças Crioulas submetidas à indução experimental de placentite, com diferentes graus de maturidade. Foram utilizados 16 potros nascidos de éguas submetidas à indução experimental de placentite, divididos em três grupos de acordo com o grau de maturidade: prematuro $(n=8)$, dismaturo $(n=4)$ e a termo $(n=4)$. Foram realizadas coletas sanguíneas nos momentos $0 \mathrm{~h}, 12 \mathrm{~h}$ e $24 \mathrm{~h}$ para realização de hemograma completo e gasometria venosa. No eritrograma, foi observada anemia normocítica normocrômica no grupo prematuro em relação ao grupo dismaturo nas $12 \mathrm{~h}$ e $24 \mathrm{~h}$. O grupo prematuro apresentou menor contagem de leucócitos totais nas $24 \mathrm{~h}$ em relação ao grupo a termo $(\mathrm{P}=0,01)$. Os valores de $\mathrm{pH}, \mathrm{cHCO}_{3}$ e $\mathrm{SO}_{2}$ não diferiram entre os grupos, porém os animais prematuros apresentaram acidose respiratória $(\mathrm{pH}=7,28)$. $\mathrm{A} \mathrm{PCO}_{2}$ nos prematuros foi maior na $0 \mathrm{~h}(\mathrm{P}=0,02)$. Nos três grupos, a $\mathrm{PCO}_{2}$ apresentou uma curva adaptativa com redução dos valores durante as $12 \mathrm{~h}$ e $24 \mathrm{~h}$. Os potros prematuros mostraram menores valores de excesso de base $(\mathrm{BE})$ no nascimento $(\mathrm{P}=0,02)$, confirmando o quadro de acidose respiratória. Concluiu-se que as respostas hematológicas e hemogasométricas diferem entre potros com diferentes graus de maturidade. A acidose observada no grupo prematuro ao nascimento, com estabilização e resposta compensatória durante as $12 \mathrm{~h}$ e $24 \mathrm{~h}$, demonstra a necessidade de avaliação hemogasométrica sequencial em potros de risco, o que permite a identificação da resposta clínica ao processo e, assim, auxilia no estabelecimento do tratamento e prognóstico para esses potros.
\end{abstract}

Palavras-chave: potros, maturidade, hematologia, gasometria, placentite ascendente

\begin{abstract}
The placentitis is a major cause of premature birth, abortion and compromised foal delivery, and may result in hypoxia and sepsis. The blood gas analysis and hematology can provide important information for monitoring the foals born from mares with placentitis, with different degrees of maturity. The aim of this study was to describe the hematological and blood gas values during the first 24 hours of life in foals born from crossbreed mares with experimentally induced placentitis, presenting different degrees of maturity. Sixteen foals, born from mares with experimentally induced ascending placentitis were assigned to three groups according to degree of maturity: premature $(n=8)$, dysmature $(n=4)$, and full-term foals $(n=4)$. Blood samples were collected at birth (0), at $12 \mathrm{~h}$ and $24 \mathrm{~h}$, and hematological evaluation and blood gas variables were measured. In the premature group normocytic normochromic anemia was observed compared to dysmature group at $12 \mathrm{~h}$ and $24 \mathrm{~h}$. The premature group showed lower count of
\end{abstract}

Recebido em 1 de julho de 2015

Aceito em 9 de agosto de 2016

*Autor para correspondência (corresponding author)

E-mail: luaraujo_sm@hotmail.com 
white blood cells at $24 \mathrm{~h}$ relative to the full-term group ( $p=0.01$ ). The $\mathrm{pH}, \mathrm{cHCO}_{3}$ and $\mathrm{SO}_{2}$ values do not differ among the groups; however the premature group showed respiratory acidosis $(p H=7,28)$. The $\mathrm{PCO}_{2}$ was higher at $\mathrm{Oh}$ in the premature foals $(p=0.02)$. In all groups, the $\mathrm{PCO}_{2}$ presented an adaptive curve with reduction between $12 \mathrm{~h}$ and $24 \mathrm{~h}$. The premature foals showed lower base excess (BE) values at birth ( $p=0.02)$, confirming the respiratory acidosis in this group. We conclude that the hematological and blood gas response differs between foals with different degrees of maturity. Acidosis in the premature foals at birth, with stabilization and compensation of $\mathrm{pH}$ value during the first 12-24h demonstrate the necessity of sequential blood gas analysis in risk foals. This may help identify the clinical response to the process and assist in the establishment of adequate treatment and prognosis for these foals.

Keywords: foals, maturity, hematology, blood gas analysis, ascending placentitis

\section{INTRODUÇÃO}

Perdas em estágio avançado da gestação e morte neonatal representam prejuízo significativo para a indústria equina (Renaudin et al., 1999). A placentite ascendente em éguas é a causa de mais de $30 \%$ dos partos prematuros e mortes neonatais dentro das primeiras $24 \mathrm{~h}$ de vida (McKinnon, 2009). A insuficiência placentária resulta em comprometimento das trocas metabólicas e gasosas entre a mãe e o feto (Marconi et al., 1999), ocasionando hipóxia fetal decorrente da diminuição do aporte de oxigênio às células. A insuficiência placentária pode, ainda, estar associada com o descolamento e o edema da placenta e a placentite (Vaala, 1999), sendo o comprometimento fetal dependente da natureza, da duração, da severidade e do estágio da gestação (Bucca, 2006).

Potros nascidos de éguas com placentite podem variar desde extremamente prematuros $(<300$ dias de gestação), com pequeno tamanho e imaturidade dos órgãos, sendo incompatível com a vida, até potros com tamanho próximo ao normal e com nenhuma ou mínimas alterações (Bain, 2004). Muitos potros podem, ainda, parecer normais ao nascimento e desenvolver sinais de comprometimento dentro das primeiras $72 \mathrm{~h}$ de vida (McAuliffe, 2008). Esses potros provenientes de éguas que apresentam insuficiência placentária devem ser considerados como potros de risco (McAuliffe, 2008) e devem ser avaliados para estabelecer a necessidade de tratamento (Bain, 2004). Segundo Giguère (2008), a avaliação e o monitoramento do potro de risco são de fundamental importância para estabelecer o prognóstico de sobrevivência, bem como o futuro atlético desses potros, e devem incluir exame dos sinais vitais, comportamento, atitude, além da utilização de métodos auxiliares, como hematologia, bioquímica sérica, gasometria e hemocultura (Morresey, 2005; Curcio e Nogueira, 2012).

A avaliação hematológica é uma ferramenta útil para o monitoramento e para guiar decisões de intervenção em casos de determinadas alterações e enfermidades. Juntamente com a hematologia, é cada vez mais utilizada a análise hemogasométrica, que fornece informações importantes sobre o estado metabólico, o equilíbrio ácido-base e da função pulmonar (Vaala, 2000), sendo útil na avaliação e no acompanhamento do quadro clínico de neonatos doentes. A hemogasometria, ou análise de gases no sangue, é um exame que tem por objetivo determinar os valores de $\mathrm{pH}$ sanguíneo, da pressão parcial de gás carbônico $\left(\mathrm{PaCO}_{2}\right)$ e oxigênio $\left(\mathrm{PaO}_{2}\right)$, íon bicarbonato $\left(\mathrm{cHCO}_{3}\right)$ e saturação de oxigênio, concentração de excesso de base (BE), entre outros, com o objetivo de avaliar principalmente o equilíbrio ácido-base orgânico.

Dessa forma, o objetivo deste estudo foi descrever os valores hematológicos e hemogasométricos avaliados durante as primeiras $24 \mathrm{~h}$ de vida em potros nascidos de éguas mestiças Crioulas com placentite ascendente induzidas experimentalmente, apresentando diferentes graus de maturidade.

\section{MATERIAL E MÉTODOS}

Este estudo foi realizado com o plantel de éguas do Centro de Ensino e Experimentação em Equinocultura da Palma (CEEP), da Universidade Federal de Pelotas, no município de Capão do Leão/RS $\left(31^{\circ} 48^{\prime} 08.2 " S\right.$; $52^{\circ} 29^{\prime} 51.4^{\prime \prime} \mathrm{O}$ ), durante a temporada reprodutiva 2012-2013. Para o estudo, foram utilizadas 16 éguas mestiças Crioulas com idade média de $11 \pm 2$ anos e média de peso de $437 \pm 22 \mathrm{~kg}$. 
As éguas foram submetidas diariamente a exame clínico geral, acompanhamento hematológico e exame obstétrico, no qual eram avaliadas as membranas placentárias, além do desenvolvimento e da atividade fetal. Todas as éguas selecionadas para este estudo não apresentavam alterações clínicas e gestacionais até o momento de indução da placentite ascendente. A indução de placentite foi realizada por meio da infusão intracervical de Streptococcus equi subespécie zooepidemicus, na concentração de $10^{7}$ UFC, entre os dias 280-295 de gestação, conforme protocolo descrito por Bailey et al. (2010). Após a indução, as éguas foram submetidas a monitoramento clínico intensivo e avaliação ultrassonográfica transretal diariamente até o momento do parto.

Todas as éguas apresentaram sinais clínicos de placentite, como secreção vulvar variando de purulenta a sanguinolenta, além de desenvolvimento precoce do úbere. No exame ultrassonográfico transretal, foi observado espessamento da junção uteroplacentária (JUP) e descolamento placentário $24 \mathrm{~h}$ a $48 \mathrm{~h}$ após a inoculação bacteriana. Nesse mesmo período pós-indução, foi identificado crescimento de $S$. equi zooepidemicus no exame microbiológico e presença de células inflamatórias na avaliação citológica, confirmando o desenvolvimento clínico de placentite.

O protocolo de tratamento das éguas foi instaurado $48 \mathrm{~h}$ após a indução de placentite e consistiu na administração de sulfatrimetoprim (Trissulfin $^{\circledR}$, sulfametoxazol e trimetoprim, Ouro Fino Agronegócio, Brasil), na dose de $30 \mathrm{mg} / \mathrm{kg}$, a cada 12 horas, durante 10 dias, e flunixin meglumine $\left(\right.$ Desflan $^{\circledR}$, Flunixin meglumine, Ouro Fino Agronegócio, Brasil), na dose de $1.1 \mathrm{mg} / \mathrm{kg}$, a cada $24 \mathrm{~h}$, com duração de sete dias.

Todos os partos foram assistidos. Após a ruptura do corioalantoide, as éguas eram encaminhadas para cocheira maternidade para acompanhamento do parto. Imediatamente após o nascimento, foi realizada avaliação clínica dos potros, com observação dos reflexos neuromusculares e comportamentais e atendimento de enfermagem ao potro neonato. O diagnóstico pós-parto de placentite foi confirmado quando na avaliação histopatológica da placenta foram observados focos de inflamação supurativa no corioalantoide e vilosidades nas regiões de estrela cervical e corpo uterino, caracterizada por presença difusa de neutrófilos, piócitos e macrófagos dispostos no corioalantoide (Hong, 1993).

Para avaliação da maturidade neonatal do presente estudo, foram considerados o tempo de gestação e a avaliação do potro mediante observação das características físicas, bem como a avaliação dos reflexos posturais e comportamentais, sendo considerado tempo para permanecer em decúbito esternal, para apresentar reflexo de sucção e para levantar.

Os potros foram divididos em três grupos: 1) prematuro, os potros com tempo gestacional menor do que 320 dias e que apresentaram características físicas de imaturidade, como baixo peso ao nascimento, pelagem macia e curta, fraco tônus muscular, com maior tempo para permanecer em estação e mamar, flacidez de orelhas e lábios, presença de cascos amolecidos (hoof pads), hiperextensão dos boletos e formato do crânio abaulado (Rossdale, 1984; Koterba, 1990; Lester, 2005); 2) dismaturo, os potros que apresentaram as características físicas de imaturidade acima descritas e que nasceram com tempo gestacional maior do que 320 dias; e 3) a termo, os potros com tempo de gestação maior do que 320 dias, com tamanho normal, pelagem longa, sem hiperextensão evidente dos boletos e expressando tempos para apresentação dos reflexos posturais e comportamentais dentro do descrito na literatura para potros saudáveis (Curcio e Nogueira, 2012). Foram incluídos oito potros no grupo prematuro, quatro potros no grupo dismaturo e quatro potros no grupo a termo.

A avaliação hematológica dos potros foi realizada considerando-se valores de contagem de hemácias, concentração de hemoglobina, hematócrito, volume corpuscular médio (VCM), concentração de hemoglobina corpuscular média $(\mathrm{CHCM})$, contagem de plaquetas, proteínas plasmáticas totais (PPT), fibrinogênio, contagem de leucócitos totais, neutrófilos, linfócitos, monócitos, eosinófilos e basófilos. As coletas de sangue foram realizadas por venopunção jugular em tubos com EDTA, imediatamente após o nascimento $(0 \mathrm{~h})$, com $12 \mathrm{~h}$ e $24 \mathrm{~h}$ de vida.

Para a análise gasométrica, amostras de sangue foram coletadas anaerobicamente, por meio de 
punção do vaso jugular, e acondicionadas em seringas heparinizadas, acondicionadas com gelo e analisadas dentro de um período máximo de uma hora após a coleta em aparelho de gasometria automático (Omni C; Roche; EUA). As variáveis analisadas foram $\mathrm{pH}$ sanguíneo, pressão parcial de oxigênio $\left(\mathrm{PO}_{2}\right)$, pressão parcial do dióxido de carbono $\left(\mathrm{PCO}_{2}\right)$, concentração de bicarbonato no plasma $\left(\mathrm{CHCO}_{3}\right.$ ), saturação de oxigênio $\left(\mathrm{SO}_{2}\right)$ e concentração de excesso de base (BE). As análises foram realizadas com $0 \mathrm{~h}, 12 \mathrm{~h}$ e $24 \mathrm{~h}$ após o nascimento.

Os dados obtidos para as variáveis dos grupos estão expressos em média \pm EPM. Todos os dados foram submetidos ao teste de normalidade de Shapiro-Wilk. As variáveis que apresentaram distribuição não paramétrica foram submetidas ao teste de Kruskal-Wallis. Para as demais variáveis na comparação entre os grupos e entre momentos, foi realizada estatística descritiva, análise de variância (General AOV) e comparação entre as médias pelo teste de LSD, com auxílio do software Statistix 9.0. Os valores de $\mathrm{P} \leq 0,05$ foram considerados significativos.

Este estudo foi aprovado pelo Comitê de Ética e Experimentação Animal da Faculdade de Veterinária da UFPel, sob o n 3891.

\section{RESULTADOS}

No eritrograma, foi observada anemia normocítica normocrômica nos potros do grupo prematuro. Foram observados menores valores na contagem de hemácias no grupo de potros prematuros em relação aos dismaturos, nos momentos $12 \mathrm{~h} \quad(8,17 \pm 0,45$ vs. $10,63 \pm 0,79$; $\mathrm{P}=0,03)$ e $24 \mathrm{~h} \quad(8,04 \pm 0,59$ vs. $10,46 \pm 0,76$; $\mathrm{P}=0,06)$. Os potros do grupo prematuro apresentaram uma redução da contagem de hemácias entre os momentos $0 \mathrm{~h}$ e $12 \mathrm{~h}$ $(10,14 \pm 0,85$ vs. $8,17 \pm 0,45 ; \mathrm{P}=0,05)$ e os animais do grupo a termo apresentaram redução entre os momentos $0 \mathrm{~h}$ e $24 \mathrm{~h}(11,17 \pm 0,58$ vs. $8,98 \pm 0,56$; $\mathrm{P}=0,06)$.

$\mathrm{Na}$ avaliação da concentração de hemoglobina, o grupo prematuro apresentou valores menores em relação aos potros dismaturos no momento $12 \mathrm{~h}$ $(10,66 \pm 0,38 \quad$ vs. $\quad 12,42 \pm 0,70 ; \quad \mathrm{P}=0,04) . \quad \mathrm{Na}$ avaliação entre momentos, todos os grupos apresentaram redução na contagem de hemoglobina entre $0 \mathrm{~h}$ e $12 \mathrm{~h}$ (prematuro:
$\mathrm{P}=0,0006$; dismaturo: $\mathrm{P}=0,009$; a termo: $\mathrm{P}=$ 0,0004). Os potros do grupo prematuro apresentaram menores valores de hematócrito em relação ao grupo dismaturo nos momentos $12 \mathrm{~h}$ $(34,27 \pm 0,99$ vs. $41,10 \pm 2,27 ; \quad \mathrm{P}=0,01)$ e $24 \mathrm{~h}$ $(33,11 \pm 2,24$ vs. $40,10 \pm 2,27 ; \mathrm{P}=0,01)$. Dentro do grupo dos prematuros, houve redução do hematócrito entre $0 \mathrm{~h}$ e $12 \mathrm{~h}(44,29 \pm 1,15$ vs. $34,27 \pm 0,99 ; \mathrm{P}=0,0001)$, e no grupo dos animais a termo houve redução dos valores entre os momentos $0 \mathrm{~h}$ e $12 \mathrm{~h}(44,92 \pm 1,46$ vs. $36,67 \pm 1,63$; $\mathrm{P}=0,0016)$.

Os valores de VCM ao nascimento, com 12 e $24 \mathrm{~h}$ nos grupos prematuro, dismaturo e a termo, foram, respectivamente: $0 \mathrm{~h} \quad(47,12 \pm 6,22$; $39,64 \pm 1,61 ; \quad 40,40 \pm 1,48), \quad 12 \mathrm{~h} \quad(42,45 \pm 1,71$; $38,87 \pm 1,46 ; 39,75 \pm 1,18)$ e $24 \mathrm{~h}(41,55 \pm 1,56$; $38,52 \pm 1,43 ; 38,97 \pm 1,33)$. Os valores de CHCM ao nascimento, com 12 e $24 \mathrm{~h}$, nos grupos prematuro, dismaturo e a termo, foram, respectivamente: $0 \mathrm{~h} \quad(30,50 \pm 0,55 ; 30,35 \pm 0,88$; $29,25 \pm 0,79), \quad 12 \mathrm{~h} \quad(31,09 \pm 0,55 ; \quad 30,25 \pm 0,49$; $29,75 \pm 0,78)$ e $24 \mathrm{~h}(31,09 \pm 0,45 ; 30,27 \pm 0,52$; $30,20 \pm 0,69)$. Não houve diferença entre grupos e momentos.

No momento do parto (0h), os potros do grupo prematuro apresentaram maiores valores de PPT em relação aos potros do grupo a termo $(5,32 \pm 0,18$ vs. $4,47 \pm 0,40 ; \mathrm{P}=0,05)$. $\mathrm{Na}$ avaliação nas $12 \mathrm{~h}$, o grupo dos dismaturos mostrou maiores valores em relação aos prematuros $(7,10 \pm 0,17$ vs. $5,81 \pm 0,25 ; \mathrm{P}=0,01)$. No momento $24 \mathrm{~h}$, os potros dismaturos apresentaram maiores valores de PPT em relação aos potros a termo $(7,00 \pm 0$ vs. $5,70 \pm 0,26 ; \mathrm{P}=0,06)$. $\mathrm{Na}$ avaliação entre momentos, somente o grupo dos animais dismaturos apresentou aumento entre os momentos $0 \mathrm{~h}$ e $12 \mathrm{~h}(4,72 \pm 0,15$ vs. $7,10 \pm 0,17$; $\mathrm{P}=0,000)$, mantendo constante nas $24 \mathrm{~h}$.

Houve redução na contagem de plaquetas entre os momentos $0 \mathrm{~h}$ e $12 \mathrm{~h} \quad(341,50 \pm 35,29$ vs. $212,25 \pm 45,09 ; \mathrm{P}=0,04)$ nos potros do grupo a termo. Os valores de fibrinogênio observados nos grupos prematuro (0h: $571,43 \pm 118,95 ; 12 \mathrm{~h}$ : $500 \pm 121.5 ;$ e $24 \mathrm{~h}: 500 \pm 75,59)$, dismaturo (0h: $466,67 \pm 66,67 ; 12 \mathrm{~h} 250 \pm 50$; e $24 \mathrm{~h}: 300 \pm 57,73) \mathrm{e}$ a termo $(0 \mathrm{~h}: 466, \overline{6} 7+176,38 ; 12 \overline{\mathrm{h}}$ : 466 , $45+170,24$; e $400+115,47)$ não apresentaram diferença entre os grupos e entre os momentos. 
Os resultados referentes aos valores de leucograma e aos valores relativos e absolutos na contagem diferencial de leucócitos estão apresentados na Tab. 1 .
Os valores de $\mathrm{pH}, \mathrm{PO}_{2}, \mathrm{PCO}_{2}$, e $\mathrm{cHCO}_{3}, \mathrm{SO}_{2}, \mathrm{BE}$ avaliados nos momentos $0 \mathrm{~h}, 12 \mathrm{~h}$ e $24 \mathrm{~h}$ estão demonstrados nas Fig. 1 e 2, respectivamente.

Tabela 1. Valores de média \pm erro-padrão (SE) para as variáveis hematológicas avaliadas durante as primeiras $24 \mathrm{~h}$ de vida nos potros prematuros, dismaturos e a termo

\begin{tabular}{|c|c|c|c|}
\hline & \multicolumn{3}{|c|}{ Momento } \\
\hline & $\begin{array}{c}\text { Oh } \\
\text { Média }+ \text { SF }\end{array}$ & $\begin{array}{c}12 \mathrm{~h} \\
\text { Médis }+\mathrm{SF}\end{array}$ & $\begin{array}{c}24 \mathrm{~h} \\
\text { Média }+\mathrm{SF}\end{array}$ \\
\hline \multicolumn{4}{|c|}{ Leucócitos totais $(\boldsymbol{\mu} \mathrm{L})$} \\
\hline Prematuros & $5500 \pm 724,08$ & $6650 \pm 1084$ & $6457 \pm 617,16^{\mathrm{B}}$ \\
\hline Dismaturos & $7125+1874,1$ & $9350+2127$ & $7050+1608,6^{\mathrm{AB}}$ \\
\hline A termo & $6850+745,54^{y}$ & $10050+735,41^{x}$ & $9400+601,39^{\mathrm{B}, \mathrm{x}}$ \\
\hline \multicolumn{4}{|c|}{ Granulócitos $(\mu \mathrm{L})$} \\
\hline Prematuros & $3088,3+716,63$ & $5080,3+1112,9$ & $4294.4+821,32$ \\
\hline Dismaturos & $4762,3+1548,9$ & $7353,3+1850,7$ & $4814,3+1505,3$ \\
\hline A termo & $5023,0+565,91$ & $7928+1070,2$ & $634, \pm 791,48$ \\
\hline \multicolumn{4}{|c|}{$\operatorname{Segmentados}(\mu \mathrm{L})$} \\
\hline Prematuros & $3071,6 \pm 50,73$ & $5073,9 \pm 1116$ & $4276,1 \pm 827,93$ \\
\hline Dismaturos & $4757,3+1553,1$ & $7353,3+1850,7$ & $4814,3+1505,3$ \\
\hline A termo & $5023+565,91^{\mathrm{y}}$ & $7928+1070,2^{\mathrm{x}}$ & $6349+791,48^{\mathrm{x}, \mathrm{y}}$ \\
\hline \multicolumn{4}{|c|}{$\operatorname{Bastonetes}(\mu \mathrm{L})$} \\
\hline Prematuros & $16,625 \pm 10,9$ & $10,125 \pm 7,10$ & $18,29 \pm 18,29$ \\
\hline Dismaturos & $5,0+\overline{5}, 0$ & - & - \\
\hline A termo & - & - & - \\
\hline \multicolumn{4}{|c|}{ Linfócitos $(\boldsymbol{\mu L})$} \\
\hline Prematuros & $2326,4+334,02$ & $1441,1+187$ & $2006,9 \pm 412,10$ \\
\hline Dismaturos & $2247,5 \pm 401,77$ & $1852,3 \pm 252,8$ & $2099,8 \pm 403,26$ \\
\hline A termo & $1744,5 \pm 180,87$ & $2001,8 \pm 345,31$ & $2798,8 \pm 560,59$ \\
\hline \multicolumn{4}{|c|}{ Monócitos $(\mu \mathrm{L})$} \\
\hline Prematuros & $62,5 \pm 18,50$ & $97,75 \pm 38,81$ & $59,43 \pm 17,36$ \\
\hline Dismaturos & $58,25 \pm 24,09$ & $86,00 \pm 51,34$ & $45,75 \pm 45,75$ \\
\hline A termo & $46,00 \pm 15,47$ & $100,25 \pm 42,68$ & $136,7 \overline{5 \pm 20,08}$ \\
\hline \multicolumn{4}{|c|}{ Eosinófilos $(\boldsymbol{\mu} \mathrm{L})$} \\
\hline Prematuros & $22,87 \pm 17,36$ & $24,12 \pm 15,92$ & $96,43 \pm 59,31$ \\
\hline Dismaturos & $57,00 \pm 35,11$ & $58,50 \pm 24,34$ & $88 \pm \overline{2} 3,65$ \\
\hline A termo & $36,50 \pm 22,19^{\mathrm{y}}$ & $20,00+20^{\mathrm{y}}$ & $115, \overline{5}+19,59^{\mathrm{x}}$ \\
\hline \multicolumn{4}{|c|}{$\begin{array}{c}\text { Relação neutrófilo:linfócito } \\
(\mathrm{N} / \mathrm{L})\end{array}$} \\
\hline Prematuros & $1,56+0,40$ & $4,34+1,26$ & $3,39+1,29$ \\
\hline Dismaturos & $1,78+0,62$ & $3,74+0,59$ & $2,57+0,64$ \\
\hline A termo & $2,87+0,03$ & $4,73+1,52$ & $2,83+0,63$ \\
\hline
\end{tabular}




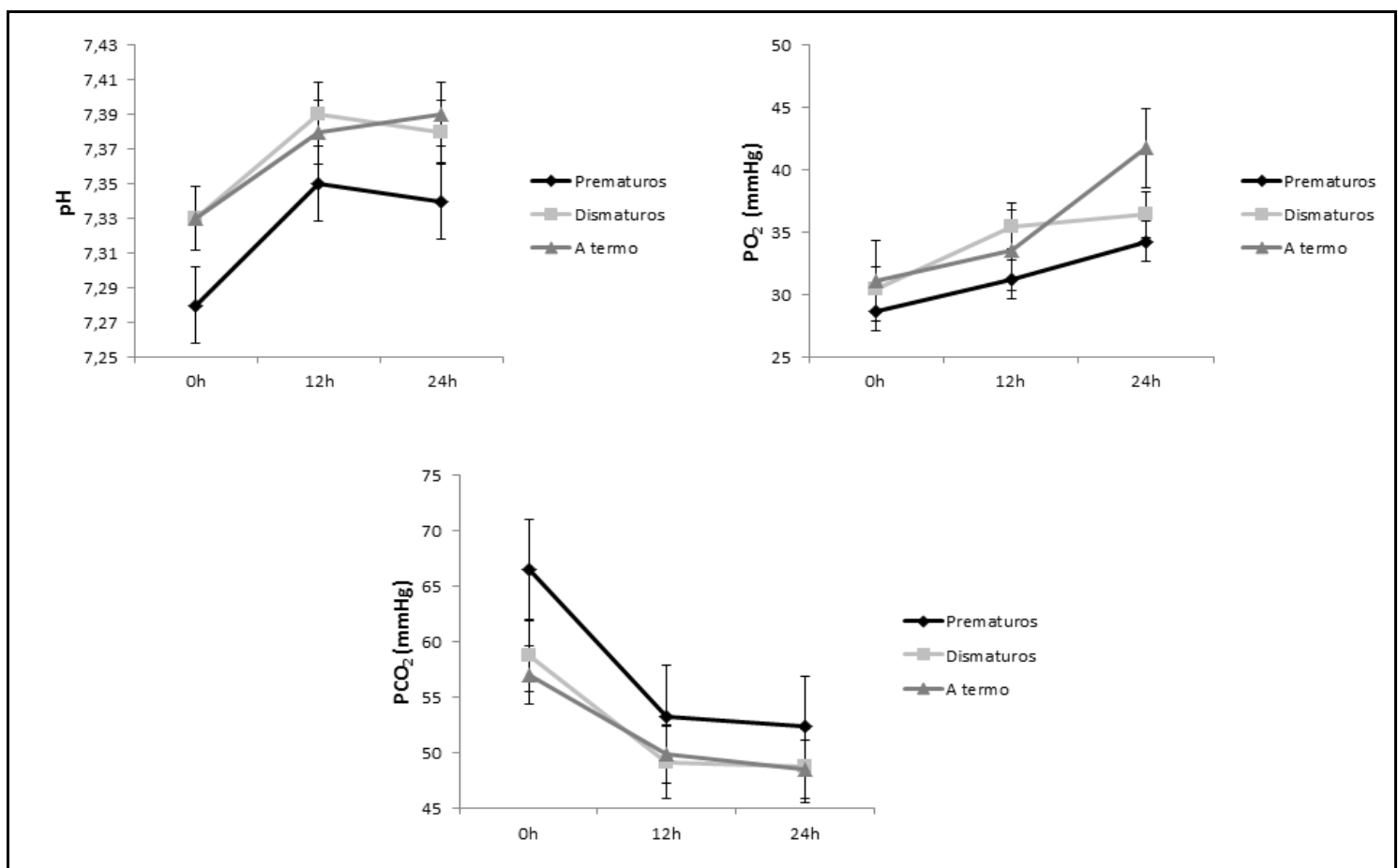

Figura 1. Mensuração dos níveis de $\mathrm{pH}$ sanguíneo, pressão parcial de oxigênio $-\mathrm{PO}_{2}$ e pressão parcial do dióxido de carbono $-\mathrm{PCO}_{2}$, em potros nascidos de éguas com placentite ascendente apresentando diferentes graus de maturidade.

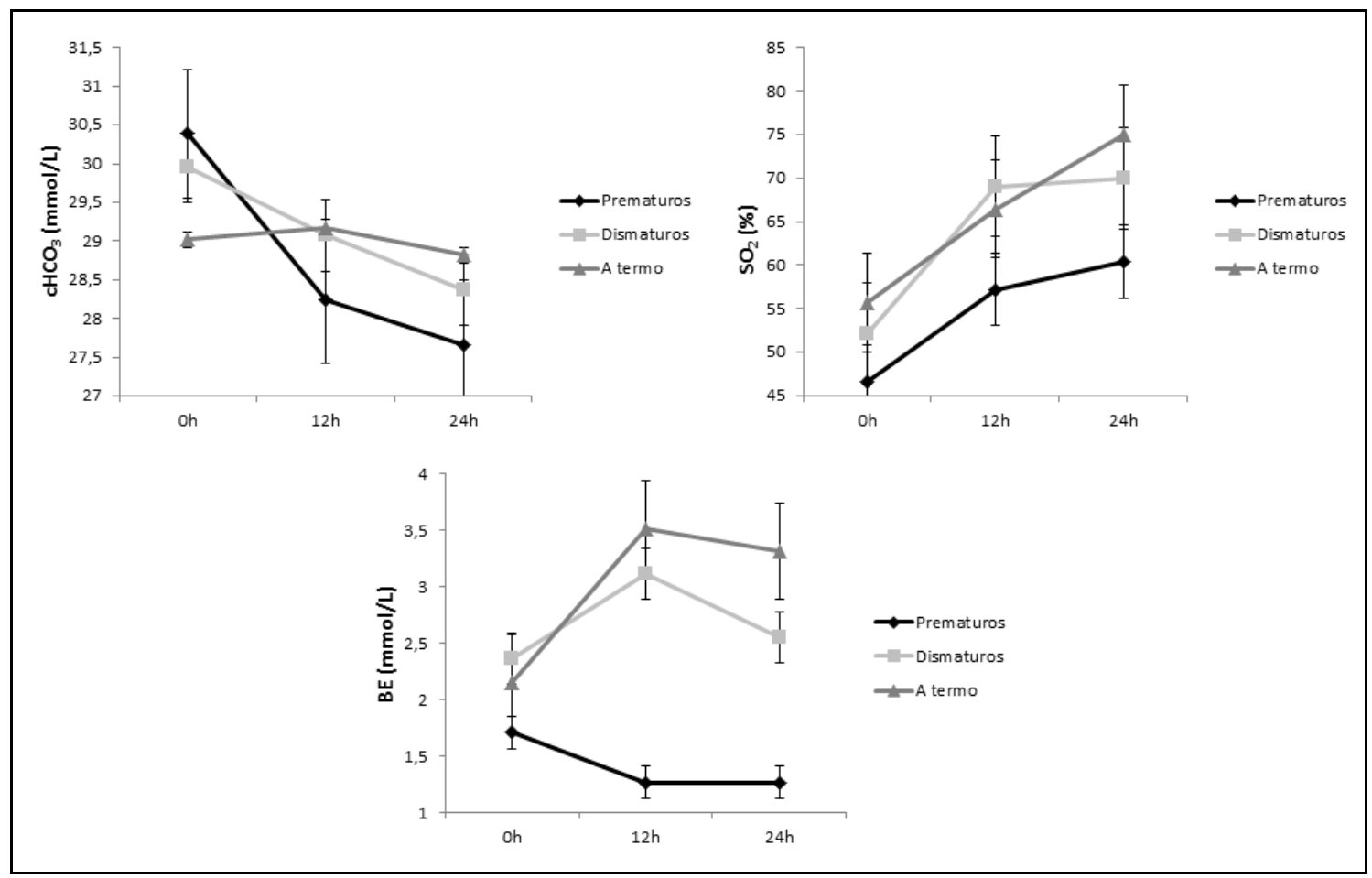

Figura 2. Mensuração dos níveis de concentração de bicarbonato no plasma - $\mathrm{cHCO}_{3}$, saturação de oxigênio $-\mathrm{SO}_{2}$ e concentração de excesso de base $-\mathrm{BE}$, em potros nascidos de éguas com placentite ascendente apresentando diferentes graus de maturidade. 


\section{DISCUSSÃO}

Neste estudo, foi observada uma redução progressiva na contagem de hemácias, hematócrito e hemoglobina durante as primeiras $24 \mathrm{~h}$ nos animais dos grupos prematuro, dismaturo e a termo, sendo considerada normal durante esse período, segundo Harvey (1990), e esse resultado é semelhante ao encontrado por Feijó et al. (2014) em um estudo com potros nascidos de éguas com placentite. Essa redução pode, em parte, ser o resultado da absorção de proteínas do colostro por pinocitose, ocasionando um aumento do volume circulante (Morresey, 2005). A análise desses marcadores não pode ser considerada um indicador sensível para o estabelecimento de um prognóstico de potros nascidos de éguas com placentite ascendente, pois tais marcadores refletem a fase de adaptação neonatal fisiológica.

Nos animais do grupo a termo, foi observado um aumento gradual dos valores de leucócitos $(6850 \pm 745,54$ vs. $10050 \pm 735,41 ; \mathrm{P}=0,025)$ e neutrófilos segmentados $(5023,0 \pm 565,91$ vs. $7928+1070,2 ; \mathrm{P}=0,009)$ entre os momentos 0h e 12h. Quando avaliado nas 24h, foram observados menores valores de leucócitos nos animais do grupo prematuro em relação aos animais do grupo a termo $(6457 \pm 617,16$ vs. 9400+601,39; $\mathrm{P}=0,01)$, concordando com o observado por Bain (2004), que descreve que potros prematuros classicamente apresentam contagem de leucócitos diminuída, porém potros provenientes de éguas com placentite, devido ao desafio prévio que sofreram, podem apresentar hemogramas mais semelhantes a potros maduros, caracterizando a importância desses parâmetros como marcadores da atividade adrenocortical, de maturidade do neonato (Rossdale, 2004).

Os valores de fibrinogênio observados não apresentaram diferenças entre os grupos e entre os momentos, mantendo-se dentro dos valores considerados normais $(100-500 \mathrm{mg} / \mathrm{dL})$ para neonatos saudáveis (Koterba, 1990). As concentrações de fibrinogênio são baixas ao nascimento $(<200 \mathrm{mg} / \mathrm{dL})$, aumentando progressivamente até o quinto mês e, então, reduzindo para próximo aos valores padrão de equinos adultos (Harvey et al., 1984). Potros que nascem com hiperfibrinogenemia, até os dois dias de idade, podem ter sofrido algum estímulo bacteriano ou inflamatório no interior do útero.
Em quadros sépticos, a hiperfibrinogenemia está associada à leucopenia e à relação N/L de 1:1 (Morresey, 2005).

Os valores de $\mathrm{pH}$ no sangue venoso não apresentaram diferença entre os grupos. Embora sem diferença, os animais do grupo prematuro apresentaram menores valores no momento $0 \mathrm{~h}$ $(\mathrm{pH}=7,28+0,02)$, caracterizando um quadro de acidemia conforme valores descritos por Gayle (1998). De acordo com Vaala (1999), em potros saudáveis, o pH em amostras arteriais logo após o nascimento é de 7,323 $\pm 0,014$. A variação de $\mathrm{pH}$ entre amostras arteriais e venosas é muito pequena, permitindo a utilização de valores de referência arteriais para amostras venosas. A acidemia presente nestes animais pode ser explicada pela infecção decorrente de placentite, pelo grau de descolamento da placenta e pela diminuição no aporte de oxigênio, resultando em asfixia, isquemia e, consequentemente, metabolismo anaeróbico. Após o momento $12 \mathrm{~h}$, todos os grupos apresentaram valores dentro dos descritos na literatura, demonstrando uma resposta compensatória e adaptativa dos potros dos grupos prematuro e dismaturo. A avaliação do $\mathrm{pH}$ sanguíneo é fundamental, pois, além dos desvios do equilíbrio ácido-base, propriamente dito, pode fornecer dados sobre a função respiratória do animal doente e sobre as condições de perfusão tecidual (Évora, 1999). A avaliação de valores de $\mathrm{pH}$ dentro dos limites normais, somente durante as primeiras $24 \mathrm{~h}$ de vida dentro do parâmetros, pode mascarar alterações iniciais que foram compensadas, como observado nos animais do grupo prematuro, reforçando a necessidade da avaliação sequencial dos valores hemogasométricos, juntamente com a evolução clínica dos animais.

Não foram observadas reduções nos valores de $\mathrm{PO}_{2}$ entre os grupos durante as primeiras 24h, que poderiam ser encontradas em decorrência de alterações placentárias, como reflexo da baixa capacidade de oxigenação pulmonar e hipoxemia (Giguère, 2008).

A $\mathrm{PCO}_{2}$ no grupo prematuro $(66,47 \pm 1,43 \mathrm{mmHg})$ foi maior em relação aos demais grupos na $0 \mathrm{~h}$, confirmando a acidose respiratória neste grupo, evidenciada pelo baixo $\mathrm{pH}$ ao nascimento. Porém, nos três grupos, observou-se uma curva adaptativa com redução dos valores de $\mathrm{PCO}_{2}$, indicando uma resposta compensatória durante 
as $12 \mathrm{~h}$ e $24 \mathrm{~h}$. Segundo Hubble (2007). elevadas concentrações da $\mathrm{PCO}_{2}$ representam ventilação alveolar inadequada, podendo ser resultado de doenças respiratórias, fadiga muscular ou patologias do SNC.

Nos animais do grupo prematuro, foram observados, embora sem diferença entre grupos, menores valores de $\mathrm{SO}_{2}$. Nos animais dos grupos dismaturos e a termo, foi observado aumento de $\mathrm{SO}_{2}$ nas $12 \mathrm{~h}$ de vida, sendo considerado normal para potros neonatos saudáveis, conforme descrito por Giguère (2008). A avaliação da $\mathrm{SO}_{2}$, segundo Corley (2002), fornece informações importantes a respeito do fornecimento de oxigênio aos tecidos e sobre a função cardiopulmonar. Diminuições na saturação de oxigênio requerem uma investigação da causa da hipoxemia ou da hipoperfusão (Corley, 2002).

Os menores valores de excesso de ácido-base (BE) observado nos animais do grupo prematuro no momento do nascimento confirmam o quadro de acidose respiratória nesses animais. A redução dos valores de $\mathrm{BE}$ entre $0 \mathrm{~h}$ e $12 \mathrm{~h}$ indica a manutenção do quadro de acidose metabólica nesses animais $(\mathrm{BE}<-2 \mathrm{mmol} / \mathrm{L})$, conforme a descrição de Rowe (2007), refletindo o grau de imaturidade e de hipóxia em decorrência da placentite.

\section{CONCLUSÃO}

Foram constatadas respostas hematológicas e hemogasométricas diferentes em relação ao grau de maturidade apresentado pelos potros. No momento do nascimento, o quadro de acidose nos animais prematuros foi confirmado mediante avaliação venosa dos valores de $\mathrm{pH}, \mathrm{PCO}_{2}$ e $\mathrm{BE}$. A estabilização desses valores durante as $24 \mathrm{~h}$ de vida demonstra a necessidade de avaliação hemogasométrica sequencial em potros de risco como ferramenta auxiliar na identificação da resposta clínica ao processo, possibilitando ao médico veterinário estabelecer o tratamento e o prognóstico para esses potros.

\section{AGRADECIMENTOS}

Os autores agradecem ao Laboratório de Análises Clínicas do Hospital Escola da Universidade Federal de Pelotas, pela realização das análises hemogasométricas, ao $\mathrm{CNPq}$ (Conselho Nacional de Desenvolvimento
Científico e Tecnológico), à Fapergs (Fundação de Amparo à Pesquisa do Estado do Rio Grande do Sul), à Capes (Coordenação de Aperfeiçoamento de Pessoal de Nível Superior) e à empresa Ouro Fino Agronegócio.

\section{REFERÊNCIAS}

BAILEY, C.S.; MACPHERSON, M.L.; POZOR, M.A. et al. Treatment efficacy of trimethoprim sulfamethoxazole, pentoxifylline and altrenogest in experimentally induced equine placentitis. Theriogenology, v.74, p.402-412, 2010.

BAIN, F.T. Management of the foal from the mare with placentitis: a clinician's approach. In: ANNUAL CONVENTION OF THE AMERICAN ASSOCIATION OF EQUINE PRACTITIONERS, 50., 2004, Denver. Proceedings... Denver: [American Association of Equine Practitioners], 2004. p.1419-1204.

BUCCA, S. Diagnosis of the compromized equine pregnancy. Vet. Clin. N. Am. Equine Pract., v.22, p.749 -761, 2006.

CORLEY, K.T.T. Monitoring and treating haemodynamic disturbances in critically ill neonatal foals. Part II: assessment and treatment. Equine Vet. Educ., v.14, p.328-36, 2002.

CURCIO, B.R.; NOGUEIRA, C.E.W. Newborn adaptations and health care throughout the first age of the foal. Anim. Reprod., v.9, p.182-187, 2012.

ÉVORA, P.R.B.; REIS, C.L.; FEREZ, M.A. et al. Distúrbios do equilíbrio hidroeletrolítico e do equilíbrio acido básico: uma revisão prática. Medicina, v.32, p.451-469, 1999.

FEIJÓ, L.S.; CURCIO, B.R.; HAETINGER, C. et al. Maturidade de potros nascidos de éguas com placentite. Arq. Bras. Med. Vet. Zootec., v.66, p.1662-1670, 2014.

GAYLE, J.M.; COHEN, N.D.; CHAFFIN, M.K. Factors associated with survival in septicemic foals: 65 cases (1988-1995). J. Vet. Intern. Med., v.12, p.140-146, 1998.

GIGUÈRE, S. Monitoring the critically ill foal. In: ANNUAL CONVENTION OF THE AMERICAN ASSOCIATION OF EQUINE PRACTITIONERS, FOCUS MEETING, 2008, Austin. Proceedings... Austin: [s.n.], 2008. p.14-22. 
HARVEY, J.W. Normal hematologic values. In: KOTERBA, A.M.; DRUMMOND, W.H.; $\mathrm{KOSCH}$, P.C. (Eds.). Equine clinical neonatology. Philadelphia: Lea \& Febiger, 1990. p.561-570.

HARVEY, J.W.; ASQUITH, R.L.; MCNULTHY, P.K. Haematology of foals up to one year old. Equine Vet. J. Suppl., v.16, p.347353,1984

HONG, C.B.; DONAHUE, J.M.; GILES, R.C. et al. Etiology and pathology of equine placentitis. J. Vet. Diagn. Invest., v.5, p.55-63, 1993.

HUBBLE, S.M.A. Acid-base and blood gas analysis. Anaesth. Intens. Care, v.7, p.427-431, 2007.

KOTERBA, A.M. Prematurity. In: KOTERBA, A.M.; DRUMMOND, W.H.; KOSCH, P.C. (Eds.). Equine clinical neonatology. Philadelphia: Lea \& Febiger, 1990. p.55-68.

LESTER, G.D. Maturity of the neonatal foal. Vet. Clin. Equine, v.21, p.333-355, 2005.

MARCONI, A.M.; PAOLINI, C.L.; STRAMARE, L. et al. Steady state maternalfetal leucine enrichments in normal and intrauterine growth-restricted pregnancies. Pediatr. Res., v.46, p.114-119, 1999.

McAULIFFE, S.B. Neonatal examination, clinical procedures and nursing care. In: McAULIFFE, S.B.; SLOVIS, N.M. (Eds.). Color atlas of diseases and disorders of the foal. Philadelphia: Saunders Elsevier, 2008. p.132165.

McKINNON, A.O. Maintenance of pregnancy. In: ANNUAL RESORT SYMPOSIUM OF THE AMERICAN ASSOCIATION OF EQUINE PRACTITIONERS, [10]., 2009, Gold Coast. Proceedings... Gold Coast: [AAFP], 2009. p.81117.
MORRESEY, P.R. Prenatal and perinatal indicators of neonatal viability. Clin. Tech. Equine Pract., v.4, p.238-249, 2005.

RENAUDIN, C.D.; TROEDSSON, M.H.T.; GILLIS, C.L. et al. Ultrasonographic evaluation of the equine placenta by transrectal and transabdominal approach in the normal pregnant mare. Theriogenology, v.47, p.559-573, 1997.

ROSSDALE, P.D. Studies in equine prematurity: guidelines for assessment of foal maturity. Equine Vet. Educ., v.16, p.300-302, 1984.

ROSSDALE, P.D. The maladjusted foal: influences of intrauterine growth retardation and birth trauma. In: ANNUAL CONVENTION OF THE AMERICAN ASSOCIATION OF EQUINE PRACTITIONERS, 50., 2004, Denver. Proceedings... Denver: Association of Equine Practitioner, 2004. p.75-126.

ROWE, K.J.; ARROWSMITH, J.E. Interpretation of measurements of arterial blood gases. Surgery, v.25, p.375-379, 2007.

VAALA, W.E. How to stabilize a critical foal prior to and during referral. In: ANNUAL CONVENTION OF THE AMERICAN ASSOCIATION OF EQUINE PRACTITIONERS, 46., 2000, San Antonio. Proceedings... San Antonio: AAEP, 2000. p.182-187.

VAALA, W.E. Peripartum Asphyxia syndrome in foals. In: ANNUAL CONVENTION OF THE AMERICAN ASSOCIATION OF EQUINE PRACTITIONERS, 1999, Albuquerque. Proceedings... Albuquerque: [AAEP], 1999. p.247-253. 